\title{
Fibrosis retroperitoneal asociada a uso crónico de ergotamina. Tratamiento con colchicina y esteroides. Caso clínico
}

\author{
JORGE VEGA ${ }^{1,2}$, HELMUTH GOECKE $^{1,2,4}$, MARIO SANTAMARINA $^{3}$
}

\section{Retroperitoneal fibrosis associated with chronic use of ergotamine. Report of one case}

\begin{abstract}
Retroperitoneal fibrosis (RPF) associated with chronic use of ergotamine is a very rare disorder. We report a 45-year-old woman who presented with a RPF after using, almost daily for 23 years, ergotamine tartrate for migraine relief. FRP presented as a chronic inflammatory state, anemia, abdominal and lumbosacral pain and a hypogastric mass. A CT-Scan showed a periaortic mass and left hydronephrosis. A percutaneous biopsy was obtained and the patient was subjected to a surgical ureterolysis and tissue resection. The biopsy confirmed the presence of RPF. Due to persistent symptoms and increase in the volume of periaortic tissue, treatment with colchicine $1 \mathrm{mg} /$ day and deflazacort $30 \mathrm{mg} /$ day was started, resulting in a rapid disappearance of symptoms, disappearance of inflammation and a significant reduction in the volume of the periaortic tissue. The patient remains in complete remission after 29 months of follow-up.
\end{abstract}

(Rev Med Chile 2011; 139: 489-494). fibrosis.

Key words: Anti inflammatory agents; Colchicine; Ergotamine; Retroperitoneal

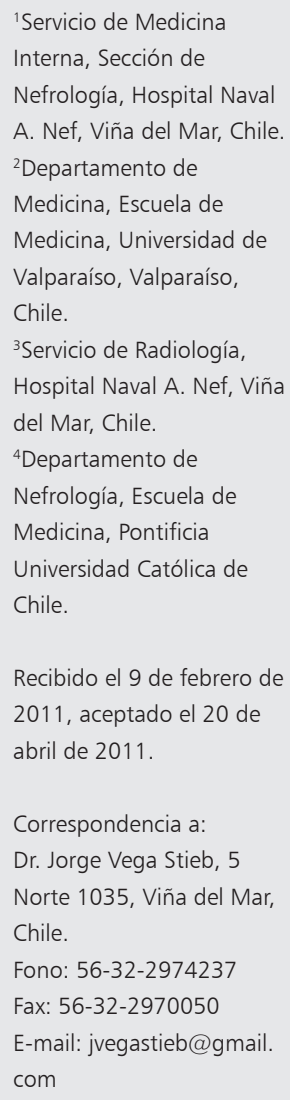

L a fibrosis retroperitoneal (FRP) es un trastorno de muy baja incidencia (5 casos por millón de habitantes) que se produce por la acumulación de un tejido inflamatorio y fibrótico en el espacio retroperitoneal, envolviendo y comprimiendo estructuras vasculares (aorta, vena cava inferior), digestivas (duodeno, vía biliar) y ureteres, pudiendo causar trastornos vasculares, obstrucción intestinal o biliar e insuficiencia renal $^{1-3}$. Su etiología es desconocida en la mayoría de los casos, pero en ocasiones puede asociarse a otros trastornos como el empleo de drogas anti-migrañosas ${ }^{4-6}$. Si bien, por su baja incidencia no existen aún estudios terapéuticos controlados en FRP, las publicaciones de casos clínicos aislados y series han comunicado la utilidad de los corticoides en altas dosis, inmunosupresores y tamoxifeno ${ }^{7}$.

Recientemente se publicó una serie clínica en que se utilizó colchicina (como agente antifibró- tico) asociada a dosis reducidas de corticoides en pacientes con FRP idiopática (FRPI) con buenos resultados, escasos efectos secundarios y ausencia de recurrencias ${ }^{8}$. En esta publicación comunicamos el uso exitoso de una combinación de colchicina y esteroides en una paciente portadora de una FRP asociada al uso crónico de ergotamina por migraña.

\section{Caso clínico}

Mujer de 45 años con historia de dolor lumbosacro e hipogástrico desde hacía meses, por lo cual había consultado a varios médicos siendo sometida a numerosos exámenes sin llegar a un diagnóstico definitivo. El dolor no se asoció a síntomas o signos propios de una enfermedad colágeno-vascular específica y los exámenes de 
autoinmunidad fueron negativos (Tabla 1). Posteriormente, un scanner abdominal mostró una masa retroperitoneal e hidronefrosis izquierda que hizo sospechar un linfoma. Se intentó instalar un catéter ureteral doble-J por vía endoscópica, lo que fue infructuoso efectuándose una nefrostomía. Meses después se realizó una biopsia por punción bajo visión con scanner de la masa retroperitoneal. La histología mostró un tejido infiltrado de tipo linfohistiocítico acompañado de fibrosis y presencia de algunos centros germinales, con un patrón morfológico de tipo reactivo. La inmunohistoquímica mostró tinción positiva para $\mathrm{CD} 3$, CD4 y bcl 2, ausencia de un patrón de restricción para lambda y kappa y tinción negativa para CD30. No se efectuó tinción para determinar la presencia de IgG4. El análisis de la muestra por 3 patólogos concluyó que existían los elementos propios de una fibrosis retroperitoneal (FRP), diagnosticando una enfermedad de Ormond. Un mes más tarde, se practicó resección parcial del tejido retroperitoneal, ureterolisis e instalación intra-operatoria de un catéter ureteral izquierdo. Un nuevo scanner efectuado un mes después de la operación mostró un tejido que se realzaba con el medio de contraste radiológico, que se iniciaba bajo la vena renal izquierda englobando a la aorta y vena cava inferior y se extendía hasta los vasos iliacos primitivos. El riñón izquierdo mostraba menor eliminación del medio de contraste que el riñón derecho y el aparato excretor estaba dilatado hasta la porción media del uréter (Figura 1). Al examen físico destacó la palpación de una masa hipogástrica mal delimitada y sensible. Los exámenes de laboratorio revelaron anemia (hemoglobina 10,9 $\mathrm{g} / \mathrm{dl}$ ), parámetros inflamatorios elevados (VHS 47 $\mathrm{mm} /$ hora, proteína C reactiva $21,3 \mathrm{mg} / \mathrm{L}$, rouleaux $+++)$, trombocitosis $\left(468.000 \mathrm{~mm}^{3}\right)$ y un perfil de autoinmunidad normal (Tabla 1). La enferma ingería desde el año anterior y a diario, tramadol, paracetamol y alprazolam para el alivio de los síntomas de su enfermedad. Al interrogatorio dirigido reconoció ingerir por auto-prescripción un medicamento para su jaqueca casi diariamente,

Tabla 1. Evolución de exámenes de laboratorio durante el tratamiento de fibrosis retroperitoneal con esteroides y colchicina

\begin{tabular}{|c|c|c|c|c|c|c|}
\hline Exámenes & Iniciales & 1 mes & 2 meses & 3 meses & 6 meses & 1 año \\
\hline Hematocrito (\%) & 33 & 37 & 39,9 & 40,8 & 38,7 & 41,6 \\
\hline Hemoglobina (g/dl) & 10,9 & 12,6 & 13,1 & 13,5 & 12,7 & 13,6 \\
\hline Leucocitos $\left(\mathrm{mm}^{3}\right)$ & 7.300 & 7.900 & 12.200 & 10.700 & 5.700 & 7.400 \\
\hline Plaquetas $\left(\mathrm{mm}^{3}\right)$ & 468.000 & 269.000 & 331.000 & 332.000 & 316.000 & 299.000 \\
\hline VHS (mm/hora) & 47 & 6 & 7 & 6 & 15 & 4 \\
\hline $\mathrm{PCR}(\mathrm{mg} / \mathrm{L})(\mathrm{VN}:<0,6)$ & 21,3 & 0,4 & $<0,3$ & 0,7 & $<0,3$ & $<0,3$ \\
\hline Creatininemia (mg/dl) & 1,06 & 1,16 & 1,07 & 0,96 & & 1,08 \\
\hline Uremia (mg/dl) & 24 & 31 & & & & \\
\hline Proteinemia (g/dl) & & & 7,0 & 6,8 & & 7,1 \\
\hline Albuminemia (g/dl) & & & 4,3 & 4,0 & & 4,3 \\
\hline Globulinas (g/dl) & & & 2,75 & 2,8 & & 2,8 \\
\hline ANA & Negativos & & & & & \\
\hline ANCA & Negativos & & & & & \\
\hline C'3 mg/dl (VN: 83-193) & 131 & & & & & \\
\hline C'4 mg/dl (VN:15-57) & 43 & & & & & \\
\hline Factor reumatoide & Negativo & & & & & \\
\hline
\end{tabular}

VHS: velocidad de eritrosedimentación; PCR: proteína C reactiva; ANA: anticuerpos antinucleares; ANCA: anticuerpos anticitoplasma de los neutrófilos; C'3: fracción 3 del complemento; C'4: fracción 4 del complemento 


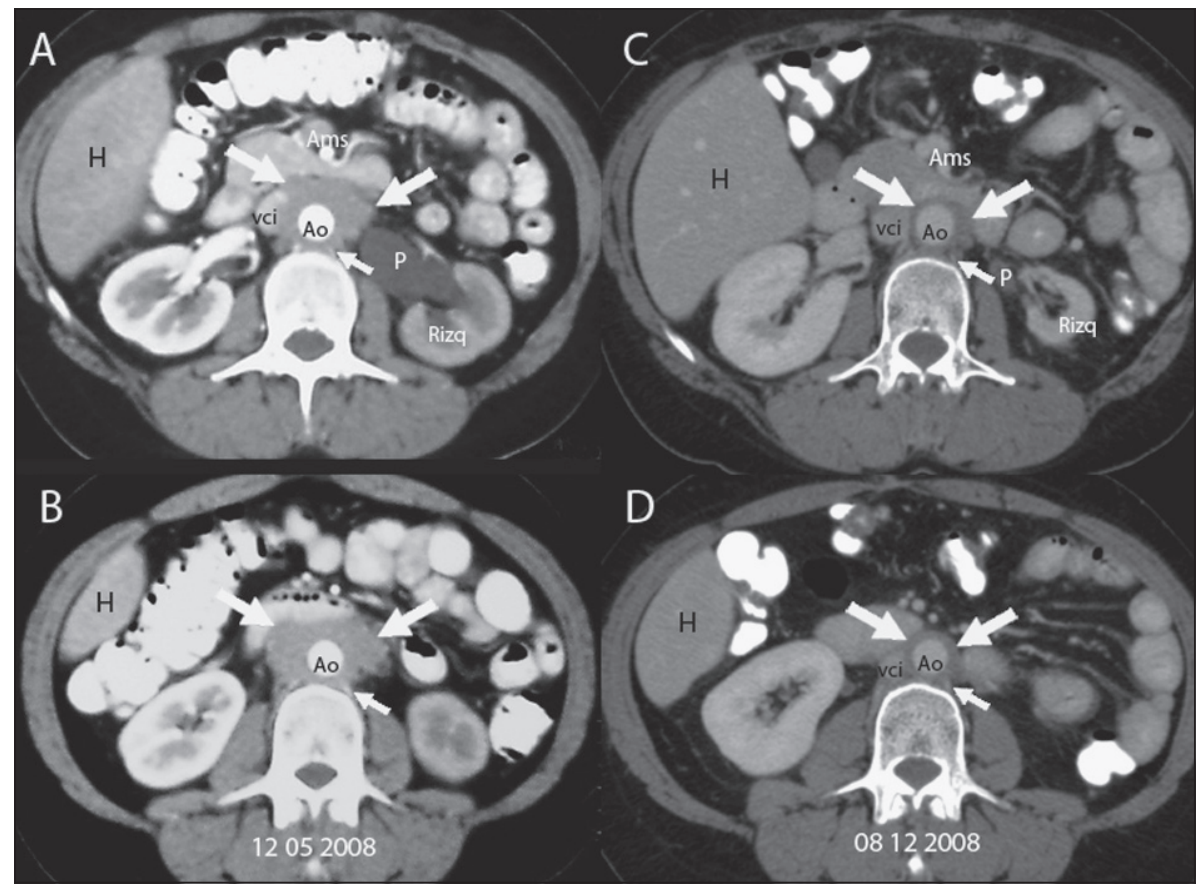

Figura 1. Imágenes axiales de TC con contraste EV en fase arterial ( $\mathrm{A}$ y B) y en fase venosa (C y D), estas últimas, efectuadas casi 6 meses después. Tanto las imágenes superiores como las inferiores, están a un mismo nivel de corte, fijado en sitio de origen de arterias lumbares (flechas blancas pequeñas). Observe que los órganos intra-abdominales y riñones no están situados a la misma altura, debido a que el nivel de inspiración del paciente, no fue el mismo. En A y B, existe la presencia de un tejido de densidad de partes blandas periaórtico (flechas blancas grandes), que rodea también en forma parcial la vena cava inferior (vci). El riñón izquierdo, presenta dilatación pielocalicial (P) y retardo en la concentración del medio de contraste endovenoso. Meses después del tratamiento (C y D), se evidencia una marcada reducción de este tejido periaórtico (flechas blancas grandes). La dilatación pielocalicial izquierda (P) ha prácticamente desaparecido, aunque el riñón izquierdo (Rizq) presenta cambios atróficos. Ao: aorta; H: hígado; AMS; arteria mesentérica superior.

desde hacía 23 años, que contenía ergotamina 1 mg y cafeína $100 \mathrm{mg}$. Con el diagnóstico de FRP asociada al uso crónico de ergotamina se inició tratamiento con colchicina $0,5 \mathrm{mg}$ cada 12 horas y deflazacort $30 \mathrm{mg} / \mathrm{día}(0,5 \mathrm{mg} / \mathrm{kg})$. En el control del mes siguiente, el dolor había disminuido significativamente, había mejorado el apetito y habían desaparecido la anemia y los signos de inflamación en los exámenes de laboratorio (Tabla 1). A los 2 meses de iniciado el tratamiento, el dolor abdominal y lumbosacro habían desaparecido completamente. Al tercer mes, estando la paciente asintomática y sin evidencias de inflamación, un nuevo scanner mostró reducción de volumen del tejido periaórtico, reducción del tamaño del riñón izquierdo y ausencia de hidronefrosis, retirándose el catéter ureteral izquierdo.

En los meses siguientes se redujo paulatina- mente la dosis de esteroides hasta llegar a una dosis de mantención de $5 \mathrm{mg}$ /día de prednisona, manteniendo la colchicina en $1 \mathrm{mg} /$ día. Un año después de iniciado el tratamiento, estando la paciente asintomática, un scanner mostró escaso tejido periaórtico y los exámenes de laboratorio ausencia de elevación de los parámetros inflamatorios. Se continuó terapia con colchicina $1 \mathrm{mg} /$ día y prednisona $5 \mathrm{mg} /$ día sin presentar síntomas de recurrencia de su enfermedad en los 29 meses de seguimiento.

\section{Discusión}

En esta paciente, la forma de presentación de la FRP fue un cuadro de compromiso del estado general de meses de evolución, fatigabilidad, dolor lumbosacro e hipogástrico y una masa hipogástri- 
ca sensible. Las pruebas de laboratorio mostraron signos de inflamación y anemia. Esta es una de las formas de presentación más frecuentes de la FRP ${ }^{1,2}$. También es común la presencia de insuficiencia renal por obstrucción ureteral bilateral ${ }^{1,2,3}$. En esta paciente la función renal era normal, pero existía hidronefrosis con disminución de la función del riñón izquierdo, que a la postre sufrió atrofia parcial.

En dos tercios de los pacientes con FRP no existe una causa evidente y se considera idiopática (FRPI). Su patogenia no es clara y solo existen teorías que intentan explicarla: reacción inflamatoria a material ateromatoso aórtico, enfermedad sistémica autoinmune, vasculitis de los vaso-vasorum aórticos, producción de anticuerpos anti-fibroblastos e infiltración por células plasmáticas $\operatorname{IgG} 4(+)$ que estimularían la fibrosis ${ }^{1,2,7,9,10}$. En el tercio restante, existen factores que pueden estar relacionados a su etiopatogenia (FRP secundarias). Estos factores son: cirugía, hemorragias, traumatismos, infecciones, tumores, radioterapia -del retroperitoneo; enfermedades autoinmunes, enfermedades esclerosantes, ateromatosis aórtica con o sin aneurisma, extravasación de orina y $\operatorname{drogas}^{1,2,3}$. De estas últimas se han mencionado los analgésicos, beta-bloqueadores, hipotensores (metildopa, hidralazina), anti-parkinsonianos (bromocriptina, pergolide), cocaína, anfetaminas y medicamentos anti-jaquecosos derivados del ergot (metisergida, tartrato de ergotamina o dihidroergotamina) $)^{6,11-20}$.

La metisergida fue retirada hace años del mercado por su asociación a FRP ${ }^{4,5}$. En una serie de 27 pacientes con FRP asociada a metisergida varios ingerían conjuntamente ergotamina. Al suspender ambas drogas los síntomas desaparecían y la hidronefrosis regresaba. Sin embargo, al reinstalar solo la ergotamina los síntomas y la obstrucción ureteral reaparecieron ${ }^{6}$. La metisergida y la ergotamina son drogas dirigidas a los receptores de serotonina y actúan como agentes vasoconstrictores potentes. La serotonina es una sustancia que promueve la fibrosis tanto a nivel experimental como en humanos, afectando al tejido pulmonar, válvulas cardíacas, endocardio, arterias y espacio retroperitoneal. Se ha planteado que estas drogas anti-jaquecosas, al unirse competitivamente a algunos receptores de serotonina, producirían un exceso de serotonina, que produciría una estimulación excesiva en los receptores no ocupados por ellas ${ }^{6}$. En pacientes portadores de tumores carcinoides, que tienen altas concentraciones de serotonina circulantes, se han descrito casos de FRP ${ }^{21,22}$.

Se ha sugerido que la vasoconstricción arteriolar prolongada, originada en el uso crónico de estas drogas en personas susceptibles, puede llevar a dilatación de las vénulas e inflamación de las mismas con exudación de un líquido rico en proteínas con la consiguiente formación de tejido fibroso ${ }^{6}$.

Se han comunicado pocos casos de FRP asociados al consumo crónico de ergotamina, a pesar de su difundido empleo en una patología tan frecuente como lo es la migraña ${ }^{6,14,17-20,23-24}$.

La terapia actual de la FRP consiste en detener la inflamación y la fibrosis y en la desobstrucción ureteral, mediante el empleo de catéteres instalados por vía endoscópica (cuando esto es posible) o mediante una nefrostomía percutánea. Años atrás, antes de disponer de técnicas endoscópicas, habitualmente se efectuaba la liberación quirúrgica de los uréteres (ureterolisis), interposición de epiplón o trasposición al espacio peritoneal ${ }^{1,2,7}$. En esta paciente, que tenía obstrucción del uréter izquierdo se intentó la colocación de un catéter ureteral por vía endoscópica, lo que no fue posible, efectuándose a una nefrostomía percutánea. Ella se mantuvo durante meses y posteriormente fue sometida a ureterolisis quirúrgica e instalación de un catéter ureteral intra-operatorio.

En las FRP asociadas a drogas anti-migrañosas se ha comunicado reducción del tejido retroperitoneal y liberación de las estructuras comprometidas al suspenderlas completamente ${ }^{6}$. Ello no ocurrió en esta paciente, a pesar de haber eliminado definitivamente el uso de ergotamina. El tejido retroperitoneal se reprodujo rápidamente después de la resección efectuada durante la ureterolisis, persistiendo un estado de inflamación crónica sintomática. En vista de la experiencia comunicada con el empleo de colchicina asociada a dosis bajas de corticoides en $\mathrm{FRPI}^{8}$, se le propuso a la paciente dicha terapia, lo que ella aceptó, ya que no deseaba utilizar dosis elevadas de esteroides ni inmunosupresores. La respuesta clínica fue rápida, con desaparición de los signos de inflamación en los exámenes de laboratorio (Tabla 1), remisión de los síntomas hasta desaparecer en el control de los 2 meses y reducción significativamente el tamaño de la masa retroperitoneal en los scanners de control (Figura 1). Ello permitió reducir la dosis de esteroides hasta una dosis de mantención de $5 \mathrm{mg} /$ día (prednisona) manteniendo la colchicina, por 
sus efectos anti-fibrótico, ahorrador de esteroides y preventivo de recurrencias ${ }^{8}$. Si bien es planteable que la buena respuesta terapéutica observada se haya debido exclusivamente a los esteroides y que la colchicina no haya tenido ningún rol, ello parece poco probable porque las dosis de corticoides empleadas en los casos comunicados de FRP habitualmente han sido muy superiores a la usada en este caso (1,0-1,5 mg/kg/día).

Recientemente se comunicó el caso de un paciente con fiebre mediterránea familiar asociada a FRI que revirtió con el uso de colchicina (indicada para el primer trastorno) sin usar esteroides. Esa comunicación apoya fuertemente la hipótesis que la colchicina puede tener por su acción antifibrótica y anti-inflamatoria, un rol terapéutico en la FRP. Los mismos autores reportaron posteriormente otros casos en que usaron exitosamente colchicina en FRPI asociada a enfermedades autoinflamatorias ${ }^{25,26}$.

En suma: comunicamos el caso de una paciente con FRP asociada al uso ergotamina, en que se utilizó un nuevo esquema terapéutico para un trastorno para el cual no existe una terapia establecida, logrando un rápido control de su enfermedad, sin efectos secundarios ni evidencias de recurrencia en un seguimiento de más de 2 años.

\section{Referencias}

1. Vaglio A, Salvarani C, Buzio C. Retroperitoneal fibrosis. Lancet 2006; 367: 241-51.

2. Van Bommel E. Retroperitoneal fibrosis. Neth J Med 2002; 60: 231-42.

3. Hemett OM, Tschopp JM, Meier P, Uldry PY, Abbet P. Retroperitoneal fibrosis: a rare cause of postrenal kidney failure, no to be missed. Rev Med Suisse 2009; 5: 226470.

4. Silberstein SD. Methysergide. Cephalalgia 1998 ;18: 42135.

5. Koehler PJ, Tfelt-Hansen PC. History of methysergide in migraine.Cephalalgia 2008; 28: 1126-35.

6. Graham JR, Suby HI, LeCompte PR, Sadowsky NL.Fibrotic disorders associated with methysergide therapy for headache. N Engl J Med 1966; 274: 359-68.

7. Swartz RD.Idiopathic retroperitoneal fibrosis: a review of the pathogenesis and approaches to treatment. Am J Kidney Dis 2009; 54: 546-53.

8. Vega J, Goecke H, Tapia H, Labarca E, Santamarina M, Martínez G.Treatment of idiopathic retroperitoneal fibrosis with colchicine and steroids: a case series. Am J Kidney Dis 2009; 53: 628-37.

9. Vaglio A, Greco P, Corradi D, Palmisano A, Martorana D, Ronda N, Buzio C.Autoimmune aspects of chronic periaortitis. Autoimmun Rev 2006; 5: 458-64.

10. Neild GH, Rodriguez-Justo M, Wall C, Connolly JO. Hyper-IgG4 disease: report and characterisation of a new disease. BMC Med 2006; 4: 23.

11. Lewis RV, McDevitt DG. Adverse reactions and interactions with beta-adrenoceptor blocking drugs. Med Toxicol 1986; 1: 343-61.

12. Sánchez-Chapado M, Angulo Cuesta J, Guil Cid M, Jiménez FJ, López Álvarez YJ. Retroperitoneal fibrosis secondary to treatment with L-dopa analogues for Parkinson disease. Arch Esp Urol 1995; 48: 979-83.

13. Ansell G. Radiological manifestations of drug-induced disease. Clin Radiol 1969; 20: 133-48.

14. Netzer P, Binek J, Hammer B. Diffuse abdominal pain, nausea and vomiting due to retroperitoneal fibrosis: a rare but often missed diagnosis. Eur J Gastroenterol Hepatol 1997; 9: 1005-8.

15. Klisnick A, Fourcade J, Ruivard M, Baud O, Souweine B, Boyer L, Deteix P. Combined idiopathic retroperitoneal and mediastinal fibrosis with pericardial involvement. Clin Nephrol 1999; 52: 51-5.

16. Sánchez-Chapado M, Angulo Cuesta J, Guil Cid M, Jiménez FJ, López Alvarez YJ. Retroperitoneal fibrosis secondary to treatment with L-dopa analogues for Parkinson disease. Arch Esp Urol 1995; 48: 979-83.

17. Moltó Ripoll F, Merenciano Cortina FJ, Herraiz Romero I, Vilas Ferrol I, Fernández-Moscoso López-Duran A, Muñoz Nuñez C, Martínez Salinas P. Retroperitoneal fibrosis due to ergotamine. Apropos a case. Arch Esp Urol 1995; 48: 400-3.

18. Damstrup L, Jensen TT. Retroperitoneal fibrosis after long-term daily use of ergotamine. Int Urol Nephrol 1986; 18: 299-301.

19. Hofstädter F. Ergotamine abuse and retroperitoneal fibrosis. Zentralbl Allg Pathol. 1976; 120: 83-7.

20. Lepage-Savary D, Vallières A. Ergotamine as a possible cause of retroperitoneal fibrosis. Clin Pharm 1982; 1: 179-80.

21. Lal A, Chen H. Treatment of advanced carcinoid tumors. Curr Opin Oncol. 2006; 18: 9-15.

22. Spivach A, Sartori A, Martinolli S, Contardo T, Zanconati F. Retroperitoneal and mesenteric fibrosis. An uncommon "carcinoid syndrome". Chir Ital 2007; 59: 565-74.

23. Martín Azcárate MD, Hernández Muro G, Isusquiza I. Retroperitoneal fibrosis secondary to ergotamine use. An Med Interna 2006; 23: 193-4. 
24. Robert M, Derbaudrenghien JP, Blampain JP, Lamy F, Meyer P. Fibrotic processes associated with long-term ergotamine therapy. N Engl J Med 1984; 311: 601-2.

25. De Socio G, Cerquaglia C, Curigliano V, Fonnesu C, Giovinale M, Verrecchia E, Marino GM, Natale L, Gasbarrini GB, Manna R. Association between familial mediterranean fever and retroperitoneal fibrosis: retro- peritoneal fibrosis regression after colchicine therapy. Int J Immunopathol Pharmacol 2009; 22: 521-4.

26. de Socio G, Verrecchia E, Fonnesu C, Giovinale M, Gasbarrini GB, Manna R. Effectiveness of colchicine therapy in 4 cases of retroperitoneal fibrosis associated with autoinflammatory diseases. J Rheumatol $2010 ; 37$ : 1971-2. 


\section{Referencias}

1. Martin W, Brynes S. Chilean Miners and Biomedical Research. A Modest Proposal. Am J Respir Crit Care Med 2010; 182: 1459-64.

2. Sobradillo P, Pozo F, Agustí A. Medicina P4: el futuro a la vuelta de la esquina. Arch Bronconeumol 2011; 47: 35-40.

Correspondencia a: Dr. Rafael Silva O.

Dos Norte 360, Talca. Fono: 71-412716 Fax: 71-209306

E-mail: rafaelsilvao@gmail.com 